( $n=22$ eyes) disease. Moreover, patients presented exophthalmos ( $n=30$ eyes), strabismus ( $n=17$ eyes), muscle fibrosis ( $n=15$ eyes) and dysthyroid optic neuropathy $(n=1)$.

TCZ was used in monotherapy $(n=27)$ or combined with methotrexate $(n=2)$ at $8 \mathrm{mg} / \mathrm{kg} / \mathrm{iv} / 4$ weeks $(\mathrm{n}=24$ ) or $162 \mathrm{mg} / \mathrm{sc} /$ week $(\mathrm{n}=5)$. TCZ yielded rapid and maintained improvement in all ocular parameters (TABLE).

After a mean follow-up of $8.96 \pm 7.55$ months using TCZ, all patients experienced ocular improvement, with TCZ withdrawal in 16 cases due to complete remission $(n=5)$ or stability of ocular inflammation $(n=11)$. Only 4 adverse effects were observed (neutropenia, external otitis, otitis media, costal osteitis).

TABLE. Improvement of ocular parameters with TCZ therapy. Data are expressed as mean \pm SD or median[IQR].

\begin{tabular}{lccccccc}
\hline & BASAL & 1 WEEK & 2 WEEKS & 1 MONTH & 3 MONTHS & 6 MONTHS & 1 YEAR \\
\hline VA & 0.7 & $0.7[0.6-$ & $0.8[0.7-1]$ & $0.85[0.7-$ & $1[0.9-1]$ & $1[0.7-1]$ & $1[0.8-1]$ \\
& {$[0.5-1]$} & $1]$ & & $1]$ & & & \\
IOP & 19.22 & 17.25 & 16.75 & 18.23 & $17.39 \pm 3.51$ & $16.52 \pm 3.56$ & 16.00 \\
& \pm 4.20 & \pm 1.90 & \pm 2.31 & \pm 5.00 & & & \pm 3.41 \\
CAS & 4.97 & 4.85 & - & $3.40 \pm 2.28$ & $2.34 \pm 1.71$ & $1.29 \pm 1.12$ & 1.11 \\
\multicolumn{7}{c}{ VA=visual acuity; IOP=intraocular pressure; CAS=clinical activity score (0/7 at baseline, $0 / 10$}
\end{tabular}

in the rest of time measures).

Conclusions: TCZ appears to be useful in TAO treatment.

REFERENCE:

[1] Bartalena L, Baldeschi L, Dickinson AJ, et al. Consensus statement of the European group on Graves' orbitopathy (EUGOGO) on management of Graves' orbitopathy. Thyroid 2008;18(3):333-346.

Disclosure of Interest: None declared

DOI: 10.1136/annrheumdis-2018-eular.4830

\section{SAT0602 CLINICAL SIGNIFICANCE OF AUTOANTIBODY POSITIVITY IN IDIOPATHIC PULMONARY FIBROSIS}

B. Ghang ${ }^{1}$, J. Lee ${ }^{1}$, O.C. Kwon¹, J.S. Oh¹, W.J. Seo ${ }^{2}$, S. Hong ${ }^{1}$, Y.-G. Kim ${ }^{1}$, B. Yoo ${ }^{1}$, J.W. Song ${ }^{3}$, C.-K. Lee ${ }^{1}$. ${ }^{1}$ Rheumatology, ASAN MEDICAL CENTER; ${ }^{2}$ Rheumatology, Seoul Veterans Hospital; ${ }^{3}$ Division of Pulmonary and Critical Care Medicine, Asan Medical Center, Seoul, Korea, Republic Of

Background: Recently, the concept of interstitial pneumonia with autoimmune features (IPAF) was proposed by the American Thoracic Society. ${ }^{1}$ The serologic domain of IPAF is composed of antibodies to extractable nuclear antigens, cyclic citrullinated peptide antibody, a high titer of rheumatoid factor, and a high titer of antinuclear antibody. However, the clinical significance of the serologic domain of IPAF has not yet been evaluated in idiopathic pulmonary fibrosis (IPF).

Objectives: To investigate the clinical significance of autoantibody positivity in IPF.

Methods: We retrospectively reviewed the records of 528 patients who met the ATS/ERS/JRS/ALAT diagnostic criteria for IPF at a tertiary hospital from January 2007 through March 2014. Patients treated with pirfenidone or nintedanib, an established IPF treatment regimen, were excluded. All patients were divided into the following 3 groups: autoimmune IPF $(n=153)$, patients with autoantibodies that met the criteria for the IPAF serologic domain; incomplete autoimmune IPF $(n=68)$, patients who did not completely meet the criteria for the IPAF serologic domain; lone IPF ( $n=307)$, patients without autoantibodies. Multivariate Cox proportional hazards models with backward elimination were used to investigate the risk factors for mortality.

Results: The 5 year mortality rates were as follows: autoimmune IPF group, $54.9 \%(n=84,3.4$ years after diagnosis [median; IQR=1.9-5.0]); incomplete autoimmune IPF group, 64.7\%; lone IPF group, 58.6\% ( $\mathrm{n}=180,2.9$ years after diagnosis [median; IQR=1.6-4.9]). Independent risk factors for mortality on multivariable analysis in the overall IPF patient population included age at diagnosis (adjusted hazard ratio $(\mathrm{HR}) 1.028, \mathrm{p}=0.002$ ), autoimmune IPF (adjusted HR 0.747, $\mathrm{p}=0.050)$, baseline $\mathrm{SaO}_{2}$ in the 6 min walking test (6MWT, adjusted HR 0.973 , $\mathrm{p}=0.012$ ), baseline distance in the 6MWT (adjusted HR 0.998, $\mathrm{p}=0.001$ ), baseline forced vital capacity (adjusted HR $0.977, \mathrm{p}<0.001$ ), and diffusing capacity of the lungs for carbon monoxide (DLCO) (adjusted HR 0.979, $\mathrm{p}<0.001$ ). In the autoimmune IPF group, use of glucocorticoid (not for management of acute exacerbation, adjusted HR 1.971, $\mathrm{p}=0.029$ ), use of immunosuppressants (adjusted HR $0.343, p=0.002$ ), baseline distance in 6MWT (adjusted HR 0.998, $p=0.032$ ), and baseline DLCO (adjusted HR 0.975, $\mathrm{p}=0.003$ ) were independent risk factors for mortality on multivariable analysis.
Abstract SAT0602 - Table 1. Risk factors associated with mortality in autoimmune IPF patients

\begin{tabular}{lcccc}
\hline \multicolumn{1}{c}{ Characteristics } & \multicolumn{4}{c}{ Multivariable analysis } \\
& Hazard & \multicolumn{2}{c}{ HR's 95\% CI } \\
& Ratios & LB & UB & P-value \\
\hline Use of glucocorticoid (not for & 1.971 & 1.073 & 3.622 & 0.029 \\
acute exacerbation management) & & & & \\
Use of immunosuppressants & 0.343 & 0.175 & 0.674 & 0.002 \\
Baseline distance in 6MWT & 0.998 & 0.996 & 1.000 & 0.032 \\
Baseline FVC & 0.984 & 0.966 & 1.002 & 0.081 \\
Baseline DLCO & 0.975 & 0.959 & 0.991 & 0.003 \\
\hline
\end{tabular}

$6 \mathrm{MWT}=6$-minute walking test; FVC $=$ forced vital capacity; DLCO $=$ diffusing capacity of the lung for carbon monoxide.

Conclusions: Our results suggest that positive autoimmunity might be a favourable factor for 5 year mortality in IPF patients compared to those without autoimmunity, and the use of immunosuppressants could be associated with improved mortality in autoimmune IPF patients.

\section{REFERENCE:}

[1] Fischer A, Antoniou KM, Brown KK, Cadranel J, Corte TJ, du Bois RM, et al. An official European Respiratory Society/American Thoracic Society research statement: interstitial pneumonia with autoimmune features. The European respiratory journal 2015;46(4):976-87.

Disclosure of Interest: None declared

DOI: 10.1136/annrheumdis-2018-eular.6020

\section{SAT0603 CAN HOMOZYGOUS OR HETEROZYGOUS MEFV MUTATIONS LEAD TO DIFFERENT PRESENTATION OF FAMILIAL MEDITERRANEAN FEVER?}

A. Aktaş ${ }^{1}$, M. Karadavut ${ }^{1}$, D. Üsküdar Cansu ${ }^{2}$, C. Korkmaz ${ }^{2} .{ }^{1}$ Internal Medicine;

${ }^{2}$ Internal Medicine, Rheumatology, Eskişehir Osmangazi University, Eskişehir,

Turkey

Background: Although some patients with familial Mediterranean (FMF) have heterozygous mutations for MEFV gene, there is a debate about whether heterozygosity for MEFV mutations in FMF might be responsible for disease symptoms or not.

Objectives: To evaluate differences between the patients with FMF having homozygos (Hom) or heterozygos (Het) MEFV mutations in terms of clinical features and severity of the disease as well as concomitant disorders.

Methods: We included 259 unrelated patients (female: 143, male: 116; mean age: $33.5 \pm 12$ years) who were clinically diagnosed as having FMF and who met the Tell-Hashomer's diagnostic criteria. The presence of MEFV mutations was investigated in exon 2,3,5 and 10 by multiplex-PCR reverse hybridization method. All clinical manifestations and their features were revised. All the patients were questioned for the presence of concurrent disorders, and the medical records of these patients were revised retrospectively. A previous diagnosis of a concomitant disease was taken into consideration if it met the relevant criteria.

Results: In 12 of 259 patient, MEFV mutation analysis was not performed. No mutation was not determined in 8 FMF patients $(3.2 \%)$. Hom mutation was found in 79 patients with FMF (31.9\%), Het plus compound heterozygotes (cHet) in 160 FMF patients (64.7\%). Early onset and early diagnosis of FMF were found in Hom group compared to Het plus cHet group (8.4 years vs 13.6 years; 23.3 years vs 28.6 years, $p<0.0001$, respectively). The number of patients with a higher severity score was significantly higher in hom group than Het plus cHet group $(p<0.001)$ No significant difference was found between Hom and Het plus cHet group in terms of clinical features except for erysipelas like erythema (ELE) $(p<0.0001)$. Concomitant disorders were as follows: ankylosing spondylitis (AS) 24 (9.3\%), amyloidosis 13 (5\%), Behcet's disease 8 (3\%). Amyloidosis (9 vs 4) was significantly higher in Hom group than Het plus cHet group $(p<0.01)$.

Conclusions: The presence of homozygous MEFV mutations in contrast to Het mutations creates a tendency for early onset of the disease, early diagnosis, frequent ELE and amyloidosis and severe disease phenotype.

Acknowledgements: None

Disclosure of Interest: None declared

DOI: 10.1136/annrheumdis-2018-eular.5062 